\title{
Determination of phenolic substances of seeds, skins and stems from white grape marc by near-infrared hyperspectral imaging
}

\author{
M.J. JARA-PALACIOS', F.J. RODRÍGUEZ-PULIDO', D. HERNANZ², M.L. ESCUDERO-GILETE' and \\ F.J. HEREDIA' \\ ' Food Colour and Quality Laboratory, Department of Nutrition and Food Science, Facultad de Farmacia, Universidad de \\ Sevilla, Sevilla 4I0I2, Spain; ${ }^{2}$ Department of Analytical Chemistry, Facultad de Farmacia, Universidad de Sevilla, Sevilla \\ 41012, Spain \\ Corresponding author: Dr Francisco J. Heredia, email: heredia@us.es
}

\begin{abstract}
Background and Aims: Winemaking generates a large quantity of grape marc that causes environmental and economic problems, which could be minimised by exploiting and adding value to these by-products. Marc is composed of seeds, skins and stems that are an important source of phenolic substances, which have antioxidant properties and potential benefits to human health. It is necessary to develop novel methods for assessing the composition of marc because conventional analyses are destructive and require lengthy preparation procedures and chemical reagents.

Methods and Results: Near-infrared hyperspectral imaging has been used for evaluating 27 individual phenolic substances in the components of grape marc after freeze-drying, the only sample preparation step. By applying partial least squares regression to the spectral data, values of $\mathrm{R}^{2}$ up to 0.98 were obtained even for estimating some minor compounds.

Conclusions: This methodology arises as an attractive alternative to analyse the phenolic composition of grape marc because of its speed and simplicity.

Significance of the Study: The use of this technique will allow wine producers to quickly determine the phenolic composition and decide the destination of this by-product.
\end{abstract}

Keywords: grape marc, hyperspectral imaging, near-infrared, phenolic composition

\section{Introduction}

Seeds, skins and stems of grapes have been recognised as an important source of phenolic substances, which have antioxidant properties and potential benefits for human health (Katalinic et al. 2010, Xia et al. 2010, Adámez et al. 2012, Anastasiadi et al. 2012). Winemaking generates a large quantity of grape marc (consisting of seeds, skins and stems) that causes environmental and economic problems, and therefore, alternative solutions to exploit and add value to these by-products are of interest because it would involve economic, social and environmental advantages (Devesa-Rey et al. 2011, Pedroza et al. 2012, Agustin-Salazar et al. 2014).

Seeds, skins and stems of grape marc exhibit a different qualitative and quantitative profile of phenolic substances, with flavanols, flavonols and phenolic acids being the main subclasses of phenolic substances in white grape marc. The concentration of these compounds is extremely variable in marc, depending on the characteristics of the grape, the winemaking process, the time of harvest and the type of by-product.

Chemical analyses, such as liquid chromatography and spectrophotometric methods, are the most widely accepted reference methods for evaluating winemaking by-products (Fontana et al. 2013). These methods, however, are frequently destructive, require lengthy preparation procedures and entail large samples. Besides, the extraction of phenolic substances is the first step in chemical analysis, requiring a long extraction time and the use of solvents, such as methanol, ethanol, propanol, acetone, ethyl acetate and their mixtures with water (Bucic-Kojic et al. 2009).
The use of these solvents involves an economic cost and sometimes creates an environmental problem. Therefore, the replacement of these conventional analyses with new methods that are nondestructive, non-time- consuming, non-solvent-consuming and roughly reliable could be of value.

Near-infrared (NIR) hyperspectral imaging is an emerging and rapid technique that provides a fast, accurate and nondestructive way to determine chemical composition (Nicolaï et al. 2007, Hernández-Hierro et al. 2012) and has been applied to several agricultural products and foods (Baye et al. 2006, Gowen et al. 2007, Sun 2010, Cubero et al. 2011, Shahin and Symons 2011, Baiano et al. 2012, Lorente et al. 2012, Hernández-Hierro et al. 2013, Nogales-Bueno et al. 2014). Hyperspectral imaging is usually carried out either in the visibleshort near-infrared (vis-NIR; 400-1000 nm) or in the NIR (1000$1700 \mathrm{~nm}$ ) spectral region (Gowen et al. 2007), and provides a digital image containing the spectrum that belongs to each pixel.

Hyperspectral images (HSI) are a three-dimensional data matrix where the first two axes of the matrix represent the spatial coordinates, whereas the third axis portrays the spectral dimension. They are presented as a battery of images where each layer shows the reflectance at a wavelength in greyscale (Burger and Geladi 2006). Hyperspectral images require the application of multivariate data analysis for data exploration because of the large amount of information that they generate.

In order to minimise contributions from imaging instrument responses that are not related to variations in the composition of the image sample, preprocessing of spectral data is often of vital 
importance if reasonable results are to be obtained from the spectral analysis step. The most frequently used methods for spectral correction are the transformation to $\log (\mathrm{l} / \mathrm{R})$ units, multiplicative scatter correction, standard normal variate (SNV) and derivation (Geladi et al. 1985, Isaksson and Naes 1988, Kaihara et al. 2002, Pizarro et al. 2004).

In contrast, spectroscopic techniques provide a large amount of data, and obtaining useful analytical information from them becomes a challenge. Because of this, it is necessary to apply multivariate statistical procedures to make the most of the data. Partial least squares regression (PLSR) is a procedure whereby a large number of independent variables (predictors) is related to one (PLSR1) or few (PLRS2) response variables (observations) when a reduced number of data are available. Because it reduces a large amount of redundant information, it is effective in spectral analysis (Lin et al. 2012, Pojic and Mastilovic 2013). Results of the leave-one-out cross-validation or test set approaches are usually compared to corroborate the prediction model results. Performance of these prediction models is evaluated using the root mean square error of calibration and cross-validation $\left(\mathrm{RMSE}_{\mathrm{C}}\right.$ and $\mathrm{RMSE}_{\mathrm{CV}}$ ), the coefficient of determination of calibration and cross-validation $\left(\mathrm{R}_{\mathrm{C}}^{2}\right.$ and $\left.\mathrm{R}_{\mathrm{CV}}{ }_{\mathrm{C}}\right)$ and number of latent variables (LV) required (Fawcett 2006).

In the wine industry it is important to know the phenolic composition of each type of by-product in each phase of harvest to determine the biofunctional potential. Besides, it is necessary to have the data quickly and accurately in order to establish the industrial applications (pharmaceutical, cosmetic, nutritional or agricultural) and to minimise the significant environmental impact. In this context, NIR hyperspectral imaging may be an option for rapidly predicting the phenolic composition of marc, without sample destruction and reagent consumption.

The aim of this study was to evaluate the potential of using the NIR hyperspectral reflectance imaging technique as a fast and non-invasive method for the determination of phenolic substances belonging to diverse phenolic groups in winemaking by-products (seeds, skins and stems) collected at several phases of harvest. This technique has been applied to grape seeds for discriminating cultivars, estimating the date of sampling and predicting phenolic composition (Rodríguez-Pulido et al. 2013, 2014), but to our knowledge, this is the first time that it has been applied to predict phenolic composition of winemaking by-products.

\section{Materials and methods}

\section{Samples}

Grape marc from the white grape Vitis vinifera cv. Zalema grown in the Condado de Huelva Designation of Origin (southwestern Spain) was used in this study. Grapes (including some stems) were harvested manually in 2012 and were processed for winemaking. After harvest, white wine was made by fermenting juice, prepared by pressing crushed grapes (with some stems) to extract all the juice. The juice was not allowed to stay in contact with the solid parts (grape marc). Grape marc is the main organic by-product generated from winemaking, which consists of a mixture of skins and pulp residues, seeds and stems. Grape marc samples, from grapes at four stages of maturation, were supplied by a winery located in the region; they were frozen immediately and stored until analysis.

Seeds, skins and stems were manually separated from the grape marc samples, and all samples $(n=12)$ were stored and further freeze-dried (lyophiliser Cryodos-80, Telstar Varian DS 102, Terrassa, Spain) for acquisition of HSI and subsequent chemical analysis. Each sample of seeds, skins and stems was divided into three parts used as replicates $(n=36)$.

\section{Hyperspectral image analysis}

Acquisition of HSI of freeze-dried samples was carried out as described by Rodríguez-Pulido et al. (2014). The system comprised a Xenics XEVA-USB InGaAs camera $(320 \times 256$ pixels; Xenics Infrared Solutions, Inc., Leuven, Belgium), a spectrograph (Specim ImSpector N17E Enhanced; Spectral Imaging Ltd, Oulu, Finland) covering the spectral range between 884 and $1717 \mathrm{~nm}$ (spectral resolution of $3.25 \mathrm{~nm}$ ), two $70 \mathrm{~W}$ tungsten iodine halogen lamps (Prilux, Barcelona, Spain) used as light source, a mirror scanner (Spectral Imaging Ltd), and a computer system. Hyperspectral images were recorded using a $50-\mathrm{Hz}$ frame rate and an exposure time of $9 \mathrm{~ms}$ using the instrument acquisition software SpectralDAQ 3.62 (Spectral Imaging Ltd). From the acquired HSI, it was observed that the first and the last 20 bands of the images had a high level of noise, and thus were not of value for spectral data extraction. Therefore, images were cropped to the spectral range of $950-1650 \mathrm{~nm}$ with a total of 215 bands.

A 'white reference' image (W, 100\% reflectance) was acquired from a white Spectralon ceramic tile (Labsphere Inc., North Sutton, NH, USA), and a 'dark reference' image (B, $0 \%$ reflectance) was obtained with the light source off and the mirror scanner completely covered with its opaque cap. The white and dark 'references' HSI were used to correct the raw images $\left(R_{0}\right)$ in order to obtain a relative reflectance image $(R)$ according to the following equation:

$$
\mathrm{R}=\frac{R_{0}-\mathrm{B}}{\mathrm{W}-\mathrm{B}} .
$$

All processes related to image and spectral analyses as well as multivariate statistical treatments were performed with the software MATLAB R2011b (The Mathworks, Natick, MA, USA).

\section{Extraction and chemical analysis}

The dry samples of seeds, skins and stems were ground to powder and then extracted with $75 \%$ methanol according to Jara-Palacios et al. (2014). The sample (1 g) was homogenised in $5 \mathrm{~mL}$ of the solvent, shaken for $1 \mathrm{~h}$ in an incubating minishaker (VWR International, Barcelona, Spain), and further centrifuged at $4190 \mathrm{~g}$ for $15 \mathrm{~min}$; the supernatant was collected and the residue submitted to the same process twice, and the supernatants combined. The extracts thus obtained were used for chemical analysis.

The individual phenolic substances were determined by rapid resolution liquid chromatography (RRLC) and MS (Jara-Palacios et al. 2014). Phenolic substances were identified by their retention time, UV-VIS spectra and mass spectra, as well as by comparison with our data library and standards when available. Proanthocyanidins, trimers, tetramers and galloyl dimers were quantified with the calibration curve of catechin. Caftaric, fertaric and coutaric acids were quantified using the calibration curves of caffeic, ferulic and coumaric acids, respectively. Quercetin derivatives were quantified as quercetin and kaempferol derivatives as kaempferol.

Total flavanols, total flavonols and total phenolic acids were also estimated by summing the concentration of each individual phenolic substance identified by RRLC. Extracts were injected three times to obtain the average, and the results were expressed as milligram phenolic substances/100 g of dry mass (DM).

\section{Results and discussion}

\section{Exploratory analysis of spectra}

The HSI of samples were acquired, calibrated and segmented in order to separate the region of interest of the background. Figure 1 shows an example of a hyperspectral image for each 
(a)

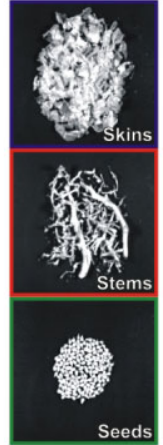

(b)

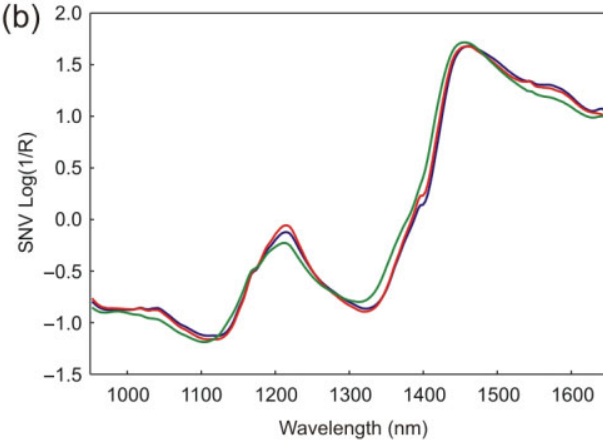

Figure 1. (a) Images belonging to the skins $(-)$, stems $(-)$ and seeds (-), components of marc at $1216 \mathrm{~nm}$, where the contrast between sample and background was the highest. (b) Average spectra of components of the marc after applying the spectral treatments.
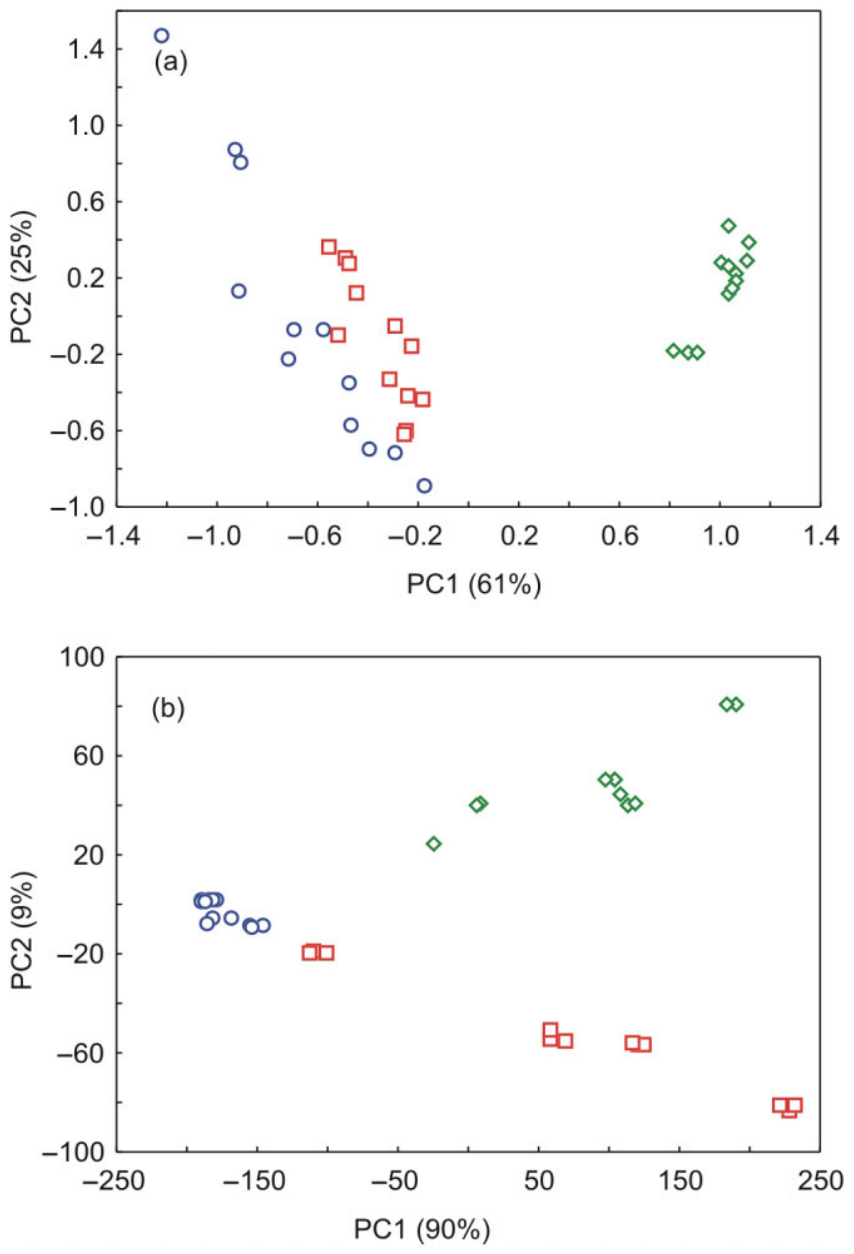

Figure 2. Scatter plots of scores for principal component 1 and principal component 2 when (a) spectral data for skins (O), stems $(\square)$ and seeds $(\diamond)$ and $(b)$ chemical data were used as independent variables in principal component analysis.

component of marc as well as their average spectra obtained after applying $\log (\mathrm{l} / \mathrm{R})$ transformation and SNV as spectral treatments. These transformations yielded the best results in the prediction models. The average spectra of seeds, skins and stems were similar, but they had different absorbance intensity along some wavelength ranges. Such likeness occurs because these three components are continuously in contact during the winemaking process. Hence, they suffer a homogenisation of composition that affects the spectra.

Table 1. Concentration of phenolic substances in the components of marc of Vitis vinifera cv. Zalema.

\begin{tabular}{|c|c|c|c|}
\hline & \multicolumn{3}{|c|}{ Concentration (mg/100g dry mass) } \\
\hline & Seeds & Skins & Stems \\
\hline Catechin & $77.3 \pm 15.8 a$ & $15.5 \pm 2.2 b$ & $68.8 \pm 19.6 a$ \\
\hline Epicatechin & $50.8 \pm 10.9 a$ & $4.3 \pm 0.4 \mathrm{~b}$ & $5.2 \pm 1.1 b$ \\
\hline Proanthocyanidin Bl & $46.8 \pm 8.7 \mathrm{a}$ & $17.1 \pm 2.7 \mathrm{~b}$ & $82.6 \pm 35.5 c$ \\
\hline Proanthocyanidin B2 & $10.8 \pm 1.4 \mathrm{a}$ & $4.8 \pm 0.3 b$ & $10.3 \pm 4.1 \mathrm{a}$ \\
\hline Proanthocyanidin B3 & $14.2 \pm 2.6 \mathrm{a}$ & $16.2 \pm 2.8 \mathrm{ab}$ & $20.0 \pm 6.3 b$ \\
\hline Proanthocyanidin B4 & $16.4 \pm 3.4 \mathrm{a}$ & $8.5 \pm 2.0 \mathrm{~b}$ & $13.9 \pm 5.0 \mathrm{a}$ \\
\hline Proanthocyanidin trimer 1 & $7.0 \pm 2.1 \mathrm{a}$ & $9.4 \pm 1.4 \mathrm{a}$ & $17.0 \pm 4.6 \mathrm{~b}$ \\
\hline Proanthocyanidin trimer 2 & $47.6 \pm 15.2 \mathrm{a}$ & $8.1 \pm 1.1 \mathrm{~b}$ & $11.5 \pm 2.0 b$ \\
\hline Proanthocyanidin tetramer 1 & $37.0 \pm 4.5 \mathrm{a}$ & $18.4 \pm 4.1 b$ & $54.7 \pm 20.7 \mathrm{C}$ \\
\hline Proanthocyanidin tetramer 2 & $13.7 \pm 1.4 \mathrm{a}$ & $7.9 \pm 3.5 b$ & $10.1 \pm 3.7 \mathrm{~b}$ \\
\hline Proanthcyanidin B2-3-O-gallate & $50.5 \pm 10.2 a$ & $11.2 \pm 2.3 b$ & $33.6 \pm 10.9 c$ \\
\hline Galloyl proanthocyanidin & $7.8 \pm 0.4 a$ & $6.4 \pm 2.5 \mathrm{a}$ & $24.8 \pm 11.3 \mathrm{~b}$ \\
\hline Total flavanols & $380 \pm 68 \mathrm{a}$ & $123 \pm 15 b$ & $351 \pm 119 a$ \\
\hline Gallic acid & $22.4 \pm 6.2 \mathrm{a}$ & $7.9 \pm 8.2 b$ & $15.5 \pm 13.1 \mathrm{ab}$ \\
\hline Protocatechuic acid & $1.0 \pm 0.2 \mathrm{a}$ & $1.3 \pm 0.6 \mathrm{a}$ & $11.5 \pm 6.6 \mathrm{~b}$ \\
\hline Caffeic acid & $0.9 \pm 0.2 \mathrm{a}$ & $2.1 \pm 0.1 b$ & $3.8 \pm 0.5 c$ \\
\hline Caftaric acid & $1.1 \pm 0.2 \mathrm{a}$ & $2.4 \pm 0.2 \mathrm{a}$ & $16.8 \pm 7.6 \mathrm{~b}$ \\
\hline cis-Coutaric acid & $0.53 \pm 0.03 a$ & $0.77 \pm 0.34 b$ & $1.18 \pm 0.17 \mathrm{c}$ \\
\hline trans-Coutaric acid & $0.75 \pm 0.04 a$ & $0.55 \pm 0.01 \mathrm{a}$ & $2.07 \pm 0.69 b$ \\
\hline Total phenolic acids & $30.1 \pm 6.3 a$ & $19.6 \pm 8.7 b$ & $74.7 \pm 14.4 \mathrm{c}$ \\
\hline Quercetin 3-O-rutinoside & $0.5 \pm 0.1 \mathrm{a}$ & $6.7 \pm 1.8 \mathrm{~b}$ & $2.3 \pm 1.4 \mathrm{c}$ \\
\hline Quercetin 3-O-glucuronide & $0.90 \pm 0.02 \mathrm{a}$ & $18.42 \pm 5.58 b$ & n.d. \\
\hline Quercetin 3-O-glucoside & $1.0 \pm 0.0 \mathrm{a}$ & $20.0 \pm 6.0 \mathrm{~b}$ & $17.0 \pm 6.3 b$ \\
\hline Quercetin pentoside & n.d. & $1.28 \pm 0.04 a$ & n.d. \\
\hline Kaempferol 3-O-galactoside & n.d. & $2.8 \pm 0.5 a$ & $1.5 \pm 0.2 b$ \\
\hline Kaempferol 3-O-glucuronide & n.d. & $1.9 \pm 0.1 \mathrm{a}$ & $1.4 \pm 0.1 b$ \\
\hline Kaempferol 3-O-glucoside & n.d. & $7.9 \pm 2.1 \mathrm{a}$ & $2.6 \pm 0.7 b$ \\
\hline Quercetin & $1.6 \pm 0.1 \mathrm{a}$ & $2.1 \pm 0.4 \mathrm{~b}$ & $2.0 \pm 0.3 \mathrm{~b}$ \\
\hline Kaempferol & n.d. & $1.36 \pm 0.02 \mathrm{a}$ & $1.55 \pm 0.06 \mathrm{~b}$ \\
\hline Total flavonols & $4.0 \pm 0.2 \mathrm{a}$ & $61.2 \pm 15.2 b$ & $27.3 \pm 8.1 \mathrm{c}$ \\
\hline
\end{tabular}

Values in the same row followed by different letters are significantly different by ANOVA test $(P<0.05)$. n.d., not detected.

Before the quantitative analysis, principal component analysis (PCA) was used as an unsupervised pattern recognition technique. Using the spectra as input data of this analysis, PCl, PC2 and PC3 explained 61, 25 and $11 \%$ of the total variance, respectively (Figure 2a). Principal component 1 had positive scores for seeds, whereas the scores for skins and stems were negative. Although the difference was weak, generally, samples of stems had higher scores than samples of skins. Figure 2a also shows how the points spread along the vertical axis (PC2). This distribution was due to the different stages of maturation of the grapes that produced the marc.

\section{Quantitative analysis}

A total of 12 flavanols, 9 flavonols and 6 phenolic acids were identified and quantified in the samples. Seeds, skins and stems exhibited a different qualitative and quantitative phenolic profile. Table 1 shows the summary of the phenolic composition for all by-products. The composition of skins, stems and seeds was statistically significantly different. The highest concentration of flavanols was found in seeds and stems $(380$ and $351 \mathrm{mg} / 100 \mathrm{~g}$ DM, respectively), whereas flavonols were more abundant in skins $(61 \mathrm{mg} / \mathrm{l00g} \mathrm{DM})$ than in stems and seeds (27 and $4 \mathrm{mg} / \mathrm{l00g} \mathrm{DM}$, respectively). In contrast, phenolic acids had the highest concentration in stems $(75 \mathrm{mg} / 100 \mathrm{~g} \mathrm{DM})$. The concentration of all the individual phenolic substances varied in the by-products; particularly proanthocyanidin B 1 , proanthocyanidin tetramer 1, proanthocyanidin B2-3-O-gallate, 
caffeic and cis-coutaric acids, quercetin 3-O-rutinoside, quercetin 3-O-glucuronide and kaempferol derivatives, which showed a significant difference between seeds, skins and stems. Besides, the phenolic composition varied between the different stages of maturation for all types of by-products (data not shown). To explore more thoroughly the latent information of the chemical results, these data were subjected to another PCA. Figure $2 \mathrm{~b}$ shows the scores for PC 1 and PC2, which explained 90 and $9 \%$ of total variance, respectively. In this case, the difference among by-products was clearer than when spectral data were used as input variables. And once again, seeds were the more isolated component in this scatter plot.

Due to the similarities of the latent information between both datasets, and in order to predict the chemical information from spectral data, a PLSR was performed. Results of phenolic composition were used as dependent $(\mathrm{Y})$ variables and the matrix of processed spectra as the independent $(\mathrm{X})$ variables in the PLSR. In order to obtain the most robust model possible, all samples were included in the PLSR model regardless of the type of by-product. The results of the prediction models for each compound are shown in Table 2.

Regression coefficients $\left(\mathrm{R}^{2}\right)$ for some individual phenolic substances extracted from marc were higher than 0.9 , the best results being $\mathrm{R}^{2}=0.98$ for kaempferol 3-O-glucoside and kaempferol 3-O-galactoside, $\mathrm{R}^{2}=0.97$ for kaempferol, and $\mathrm{R}^{2}=0.96$ for epicatechin and trans-coutaric acid. In these cases, RMSE $_{\mathrm{CV}}$ were $0.41,0.11,0.02,4.71$ and $0.16 \mathrm{mg} / 100 \mathrm{~g} \mathrm{DM}$, respectively, which involved an error of prediction around $13 \%$. The results for predicting the families of compounds, that is total flavanols, total phenolic acids and total flavonols, were $\mathrm{R}^{2}=0.78$, 0.87 and 0.70 , respectively. The RMSE $E_{C v}$ was $66.63 \mathrm{mg} / 100 \mathrm{~g} \mathrm{DM}$ for total flavanols, $14.27 \mathrm{mg} / 100 \mathrm{~g}$ DM for total flavonols and $9.61 \mathrm{mg} / 100 \mathrm{~g}$ DM for total phenolic acids.

\section{Conclusions}

Samples of the components of grape marc were successfully evaluated by PLSR models; up to 27 individual phenolic substances were quantified in seeds, stems and skins by this method. Although freeze-drying was necessary to obtain reliable results, the technique required no reagent and no extraction, and thus was more environmentally friendly than other techniques such as chromatography. The composition of agricultural products is variable; therefore, when future samples are analysed, it will be necessary to evaluate other factors that may influence the prediction models. Therefore, a comprehensive study must be made in order to evaluate other factors, such as production area, vintage or cultivar, for the complete

Table 2. Mean, minimum and maximum concentration of phenolic substances, number of latent variables, calibration and cross-validation results for the PLSR models obtained from processed spectra.

\begin{tabular}{|c|c|c|c|c|c|c|c|c|}
\hline & \multicolumn{8}{|c|}{ Concentration (mg/100g dry mass) } \\
\hline & Mean & Minimum & Maximum & \#LV & $\mathrm{RMSE}_{\mathrm{C}}$ & $\mathbf{R}^{2}{ }_{C}$ & $\mathbf{R M S E}_{\mathrm{CV}}$ & $\mathbf{R}^{2}{ }_{\mathrm{CV}}$ \\
\hline Catechin & 53.86 & 11.33 & 104.11 & 6 & 10.34 & 0.89 & 14.00 & 0.80 \\
\hline Epicatechin & 23.17 & 3.90 & 66.56 & 4 & 3.70 & 0.97 & 4.72 & 0.96 \\
\hline Proanthocyanidin Bl & 48.81 & 13.89 & 130.88 & 6 & 14.67 & 0.81 & 20.53 & 0.65 \\
\hline Proanthocyanidin B2 & 8.99 & 3.80 & 15.10 & 6 & 1.29 & 0.87 & 1.86 & 0.75 \\
\hline Proanthocyanidin B3 & 16.83 & 8.51 & 32.40 & 6 & 2.60 & 0.70 & 3.43 & 0.50 \\
\hline Proanthocyanidin B4 & 12.94 & 6.69 & 24.05 & 5 & 2.26 & 0.78 & 3.01 & 0.63 \\
\hline Proanthocyanidin trimer 1 & 11.16 & 2.72 & 23.95 & 6 & 2.28 & 0.80 & 3.12 & 0.65 \\
\hline Proanthocyanidin trimer 2 & 22.39 & 2.72 & 58.60 & 2 & 7.01 & 0.87 & 7.68 & 0.86 \\
\hline Proanthocyanidin tetramer 1 & 36.71 & 13.73 & 84.01 & 6 & 8.47 & 0.80 & 11.62 & 0.65 \\
\hline Proanthocyanidin tetramer 2 & 10.57 & 5.14 & 17.30 & 6 & 1.93 & 0.74 & 2.66 & 0.53 \\
\hline Proanthcyanidin B2-3-O-gallate & 31.76 & 8.59 & 63.99 & 5 & 4.86 & 0.93 & 6.29 & 0.89 \\
\hline Galloyl proanthocyanidin & 13.59 & 3.91 & 42.73 & 6 & 4.90 & 0.79 & 7.27 & 0.58 \\
\hline Total flavanols & 285.05 & 109.55 & 498.63 & 6 & 48.03 & 0.88 & 66.63 & 0.78 \\
\hline Gallic acid & 15.27 & 2.57 & 37.32 & 6 & 4.14 & 0.86 & 5.58 & 0.75 \\
\hline Protocatechuic acid & 4.91 & 0.58 & 19.59 & 4 & 2.14 & 0.88 & 2.70 & 0.82 \\
\hline Caffeic acid & 2.27 & 0.74 & 4.19 & 6 & 0.25 & 0.96 & 0.36 & 0.92 \\
\hline Caftaric acid & 6.76 & 0.85 & 25.91 & 6 & 2.12 & 0.94 & 2.56 & 0.91 \\
\hline cis-Coutaric acid & 0.82 & 0.49 & 1.39 & 6 & 0.11 & 0.90 & 0.15 & 0.83 \\
\hline trans-Coutaric acid & 1.31 & 0.54 & 2.77 & 6 & 0.13 & 0.97 & 0.19 & 0.95 \\
\hline Total phenolic acids & 41.46 & 13.57 & 98.93 & 6 & 7.04 & 0.92 & 9.61 & 0.87 \\
\hline Quercetin 3-O-rutinoside & 3.18 & 0.33 & 9.62 & 5 & 1.41 & 0.76 & 1.82 & 0.63 \\
\hline Quercetin 3-O-glucuronide & 9.66 & 0.86 & 26.90 & 6 & 2.82 & 0.91 & 4.36 & 0.81 \\
\hline Quercetin 3-O-glucoside & 12.64 & 0.93 & 29.15 & 6 & 4.40 & 0.79 & 5.95 & 0.64 \\
\hline Quercetin pentoside & 1.28 & 1.26 & 1.39 & 4 & 0.01 & 0.94 & 0.04 & 0.15 \\
\hline Kaempferol 3-O-galactoside & 2.18 & 1.35 & 3.78 & 5 & 0.07 & 0.99 & 0.11 & 0.98 \\
\hline Kaempferol 3-O-glucuronide & 1.76 & 1.31 & 2.10 & 5 & 0.04 & 0.97 & 0.07 & 0.93 \\
\hline Kaempferol 3-O-glucoside & 5.26 & 2.02 & 11.62 & 5 & 0.27 & 0.99 & 0.41 & 0.98 \\
\hline Quercetin & 1.92 & 1.45 & 2.84 & 4 & 0.16 & 0.79 & 0.19 & 0.72 \\
\hline Kaempferol & 1.50 & 1.34 & 1.64 & 4 & 0.01 & 0.99 & 0.02 & 0.97 \\
\hline Total flavonols & 30.83 & 3.73 & 83.65 & 6 & 10.56 & 0.82 & 14.27 & 0.70 \\
\hline
\end{tabular}

Values having $\mathrm{R}^{2}$ Cv greater than 0.90 are shown in bold. LV, latent variable; PLSR, partial least squares regression; $\mathrm{R}_{\mathrm{C}}$

$\mathrm{R}^{2}{ }_{\mathrm{CV}}$, coefficient of determination of cross-validation; $\mathrm{R}_{\mathrm{C}}^{2}$, coefficient of determination of cross-validation; $\mathrm{RMSE}_{\mathrm{C}}$, root mean square error of calibration; $\mathrm{RMSE} \mathrm{CV}_{\mathrm{C}}$, root mean square error of cross-validation. 
development of these models. Although it is not a substitute for conventional chemical analysis, it is an attractive alternative due to its speed and simplicity. By controlling all the possible variables that affect each cultivar, this could become a reference method to assess the chemical composition of grape marc for researchers, and may be useful for deciding the destination of this by-product in wineries.

\section{Acknowledgements}

The authors acknowledge the collaboration of Vinícola del Condado winery (Condado de Huelva DO) for supplying samples and the assistance of the technical staff of Biology Service (SGI, Universidad de Sevilla). Dr M.J. Jara-Palacios holds a predoctoral Research Grant (FPU) from the Spanish Ministry of Education. Dr F.J. Rodríguez-Pulido also thanks VPPI-Universidad de Sevilla for a postdoctoral grant. Authors thank the Ministerio de Economía y Competitividad, Gobierno de España, the financial support (Project AGL2014-58486-C22-R).

\section{References}

Adámez, J.D., Samino, E.G., Sánchez, E.V. and González-Gómez, D. (2012) In vitro estimation of the antibacterial activity and antioxidant capacity of aqueous extracts from grape-seeds (Vitis vinifera L.). Food Control 24, 136-141.

Agustin-Salazar, S., Medina-Juárez, L.A., Soto-Valdez, H., Manzanares-López, F. and Gámez-Meza, N. (2014) Influence of the solvent system on the composition of phenolic substances and antioxidant capacity of extracts of grape (Vitis vinifera L.) marc. Australian Journal of Grape and Wine Research 20, 208-213.

Anastasiadi, M., Pratsinis, H., Kletsas, D., Skaltsounis, A. and Haroutounian, S. (2012) Grape stem extracts: polyphenolic content and assessment of their in vitro antioxidant properties. LWT-Food Science and Technology 48, 316-322.

Baiano, A., Terracone, C., Peri, G. and Romaniello, R. (2012) Application of hyperspectral imaging for prediction of physico-chemical and sensory characteristics of table grapes. Computers and Electronics in Agriculture 87, 142-151.

Baye, T.M., Pearson, T.C. and Settles, A.M. (2006) Development of a calibration to predict maize seed composition using single kernel near infrared spectroscopy. Journal of Cereal Science 43, 236-243.

Bucic-Kojic, A., Planinic, M., Tomas, S., Jakobek, L. and Seruga, M. (2009) Influence of solvent and temperature on extraction of phenolic compounds from grape seed, antioxidant activity and colour of extract. International Journal of Food Science and Technology 44, 2394-2401.

Burger, J. and Geladi, P. (2006) Hyperspectral NIR imaging for calibration and prediction: a comparison between image and spectrometer data for studying organic and biological samples. The Analyst 131, 1152-1160

Cubero, S., Aleixos, N., Moltó, E., Gómez-Sanchis, J. and Blasco, J. (2011) Advances in machine vision applications for automatic inspection and quality evaluation of fruits and vegetables. Food and Bioprocess Technology 4, 487-504.

Devesa-Rey, R., Vecino, X., Varela-Alende, J.L., Barral, M.T., Cruz, J.M. and Moldes, A.B. (2011) Valorization of winery waste vs. the costs of not recycling. Waste Management 31, 2327-2335.

Fawcett, T. (2006) An introduction to ROC analysis. Pattern Recognition Letters 27, 861-874.

Fontana, A.R., Antoniolli, A. and Bottini, R.J. (2013) Grape pomace as a sustainable source of bioactive compounds: extraction, characterization, and biotechnological applications of phenolics. Journal of Agricultural and Food Chemistry 61, 8987-9003.

Geladi, P., MacDougall, D. and Martens, H. (1985) Linearization and scattercorrection for near-infrared reflectance spectra of meat. Applied Spectroscopy 39, 491-500.

Gowen, A.A., O’Donnell, C.P., Cullen, P.J., Downey, G. and Frias, J.M. (2007) Hyperspectral imaging - an emerging process analytical tool for food quality and safety control. Trends in Food Science \& Technology 18, $590-598$.
Hernández-Hierro, J.M., Valverde, J., Villacreces, S., Reilly, K., Gaffney, M., González-Miret, M.L., Heredia, F.J. and Downey, G. (2012) Feasibility study on the use of visible-near-infrared spectroscopy for the screening of individual and total glucosinolate contents in broccoli. Journal of Agricultural and Food Chemistry 60, 7352-7358.

Hernández-Hierro, J.M., Nogales-Bueno, J., Rodríguez-Pulido, F.J. and Heredia, F.J. (2013) Feasibility study on the use of near-infrared hyperspectral imaging for the screening of anthocyanins in intact grapes during ripening. Journal of Agricultural and Food Chemistry 61, 9804-9809.

Isaksson, T. and Næs, T. (1988) The effect of multiplicative scatter correction (MSC) and linearity improvement in NIR spectroscopy. Applied Spectroscopy 42, 1273-1284.

Jara-Palacios, M.J., Hernanz, D., González-Manzano, S., Santos-Buelga, C., Escudero-Gilete, M.L. and Heredia, F.J. (2014) Detailed phenolic composition of white grape by-products by RRLC/MS and measurement of the antioxidant activity. Talanta 125, 51-57.

Kaihara, M., Takahashi, T., Akazawa, T., Sato, T. and Takahashi, S. (2002) Application of near infrared spectroscopy to rapid analysis of coals. Spec troscopy Letters 35, 369-376.

Katalinic, V., Mozina, S.S., Skroza, D., Generalic, I., Abramovic, H., Miloš, M., Ljubenkov, I., Piskernik, S., Pezo, I., Terpinc, P. and Boban, M. (2010) Polyphenolic profile, antioxidant properties and antimicrobial activity of grape skin extracts of 14 Vitis vinifera varieties grown in Dalmatia (Croatia). Food Chemistry 119, 715-723.

Lin, P., Chen, Y. and He, Y. (2012) Identification of geographical origin of olive oil using visible and near-infrared spectroscopy technique combined with chemometrics. Food and Bioprocess Technology 5, 235-242.

Lorente, D., Aleixos, N., Gómez-Sanchis, J., Cubero, S., García-Navarrete, O. and Blasco, J. (2012) Recent advances and applications of hyperspectral imaging for fruit and vegetable quality assessment. Food and Bioprocess Technology 5, 1121-1142

Nicolaï, B.M., Beullens, K., Bobelyn, E., Peirs, A., Saeys, W., Theron, K.I. and Lammertyn, J. (2007) Nondestructive measurement of fruit and vegetable quality by means of NIR spectroscopy: a review. Postharvest Biology and Technology 46, 99-118.

Nogales-Bueno, J., Hernández-Hierro, J.M., Rodríguez-Pulido, F.J. and Heredia, F.J. (2014) Determination of technological maturity of grapes and total phenolic compounds of grape skins in red and white cultivars during ripening by near infrared hyperspectral image: a preliminary approach. Food Chemistry 152, 586-591.

Pedroza, M.A. Carmona, M., Pardo, F. Salinas, M.R and Zalacain, A. (2012) Waste grape skins thermal dehydration: potential release of colour, phenolic and aroma compounds into wine. CyTA-Journal of Food 10 225-234.

Pizarro, C., Esteban-Díez, I., Nistal, A.J. and González-Sáiz, J.M. (2004 Influence of data pre-processing on the quantitative determination of the ash content and lipids in roasted coffee by near infrared spectroscopy. Analytica Chimica Acta 509, 217-227.

Pojic, M.M. and Mastilovic, J.S. (2013) Near infrared spectroscopyadvanced analytical tool in wheat breeding, trade, and processing. Food and Bioprocess Technology 6, 330-352.

Rodríguez-Pulido, F.J., Barbin, D.F., Sun, D.W., Gordillo, B., González-Miret, M.L. and Heredia, F.J. (2013) Grape seed characterization by NIR hyperspectral imaging. Postharvest Biology and Technology $\mathbf{7 6}$ 74-82.

Rodríguez-Pulido, F.J., Hernández-Hierro, J.M., Nogales-Bueno, J., Gordillo, B., González-Miret, M.L. and Heredia, F.J. (2014) A novel method for evaluating flavanols in grape seeds by near infrared hyperspectral imaging. Talanta 122, 145-150.

Shahin, M.A. and Symons, S.J. (2011) Detection of Fusarium damaged kernels in Canada Western Red Spring wheat using visible/near-infrared hyperspectral imaging and principal component analysis. Computers and Electronics in Agriculture 75, 107-112.

Sun, D.W. (2010) Hyperspectral imaging for food quality analysis and control (Elsevier Science and Technology: San Diego, CA, USA).

Xia, E.Q., Deng, G.F., Guo, Y.J. and Li, H.B. (2010) Biological activities of polyphenols from grapes. International Journal of Molecular Sciences 11, 622-646. 